\title{
Recursos das tecnologias de informação e comunicação mediando o ensino- aprendizagem e configurando ecologias cognitivas de estudantes do Centro de Ciências Rurais
}

\author{
Liziany Müller - NTE - Universidade Federal de Santa Maria \\ lizianym@ hotmail.com
}

Andriéli Hedlund Bandeira - PEG/CE - Universidade Federal de Santa Maria andrieli_hedlund@ hotmail.com

Bruna Mendonça Alves - PEG/CE - Universidade Federal de Santa Maria brunamalves_11@ hotmail.com

Cláudia Smaniotto Barin - NTE - Universidade Federal de Santa Maria claudiabarin@ cead.ufsm.br

Elena Maria Mallmann - CE - Universidade Federal de Santa Maria elena@nte.ufsm.br

\section{Resumo}

Este trabalho teve por objetivo investigar a contribuição de práticas pedagógicas que integram recursos das tecnologias de informação e comunicação na mediação do processo ensino-aprendizagem para configuração de ecologias cognitivas. A metodologia foi pautada em uma pesquisa exploratória num paradigma construtivista. Como instrumento de coleta de dados foi realizado questionário tipo survey com estudantes dos Cursos de Graduação em Agronomia, Tecnologia de Alimentos e Medicina Veterinária da Universidade Federal de Santa Maria, RS, Brasil. A integração de recursos das tecnologias de informação e comunicação modifica a concepção de ensino-aprendizagem gerando comunicação mais contínua e sensorial. Além da verbalização, a hipermídia potencializa interatividade que produz interação e criação de conhecimento. O processo ensino-aprendizagem mediado por hipermídia permite aliar o conhecimento e a formação epistemológica à integração reflexiva, pedagógica, coletiva, articuladora e atrativa dos recursos configurando a ecologia cognitiva. Assim, a interatividade resignifica a relação indivíduo-objeto criando uma nova dinâmica nos processos de construção do saber baseados na existência de relações, diálogos e interações.

Palavras-chave: tecnologia Educacional; TIC; ensino-aprendizagem; hipermídia.

\section{Technology resources for information and communication mediated learning and teaching-cognitive ecology setting of students of Rural Sciences Center}

\begin{abstract}
This study aimed to investigate the effect of pedagogical practices that use the resources of Information and Communication Technology (ICT) in mediating the teaching-learning process and configuration of students' cognitive ecologies. The methodology used was based on an exploratory qualitative approach with a constructivist paradigm. We interviewed students of undergraduate programs in Agronomy, Veterinary Medicine and Food Technology, Federal University of Santa Maria, RS, Brazil. As data collection instrument was used a questionnaire type survey for students. The use of ICT changes the conception of the individual allowing him not to limit, but rather dare, communication becomes more continuous and sensory, because, beyond words hypermedia becomes a new means of conveying information to potential knowledge. The teaching-learning mediated by ICT allows to combine the knowledge and training to use reflective epistemological, pedagogical, collective, and
\end{abstract}


attractive organizer of resources, setting the students' cognitive ecology, providing thus a redefinition of individual and object, creating a new dynamic in the process of knowledge construction based on the existence of relations, dialogue and collective interactions.

Keywords: Resources; Educational Technology; ICTs; Teaching and learning.

\section{Introdução}

Nas últimas décadas, os debates sobre os efeitos das contínuas e rápidas transformações da sociedade contemporânea no contexto escolar se ampliam constantemente, pois as relações entre estudantes e professores mudaram tornando-se mais horizontais e sem lugar fixo (Pessanha, 2009).

As tecnologias da informação e comunicação são tecnologias da inteligência, por serem novas ferramentas cognitivas que possibilitam um salto qualitativo em nossas possibilidades de raciocínio e apreensão de conhecimento (Lévy, 1993). No cotidiano, a integração das Tecnologias de Informação e Comunicação (TIC) criou novas formas de interação entre as pessoas, que implicaram no redimensionamento das funções e dos papéis sociais.

As relações sociais passaram a ser mediadas pelo computador, independentes de espaço e tempo definidos (Morije; Pavan, 2004). A facilidade de acesso às redes, tendem a tornar a gerar mais autonomia, embora muitos ainda não descobriram as potencialidades da mediação tecnológica, o que abre um novo espaço para o trabalho informacional de promover, capacitar, organizar e prover o acesso à esses novos recursos (Gonçalves, 1998).

As TIC vêm sendo utilizadas para agilizar, remodelar e complementar as formas de comunicação que eram usadas antes delas existirem (Alves; Nova, 2003). Perrenoud (2000, p. 128) chama atenção para a importância de se formar para as TIC:

Formar para novas tecnologias é formar o julgamento, o senso crítico, hipotético e dedutivo, as faculdades de observação e de pesquisa, a imaginação, a capacidade de memorizar e classificar, a leitura e análise de texto e imagens, a representação de redes, de procedimentos e de estratégias de comunicação.

Os profissionais da educação, com a chegada das tecnologias nas escolas, deparam-se como o desafio de problematizá-las e utilizá-las pedagogicamente no processo ensino-aprendizagem. Como a educação não pode abster-se dos recursos das TIC, é necessário inovar em termos de atuação dos professores e gestores. Simões (2002) ressalta a necessidade da escola rever o seu projeto pedagógico, reconhecendo de forma crítica e adequada a presença das tecnologias na vivência do estudante fora do contexto escolar.

A utilização dos recursos das TIC no processo ensino-aprendizagem poderá contribuir para que os estudantes superem as dificuldades de aprendizagem $\mathrm{e}$ internalizem o conteúdo ensinado (Mortimer; Scott, 2002). Logo no início da integração dos recursos das TIC na escola, imaginou-se que era a solução dos problemas educacionais, podendo chegar inclusive a substituir os professores. Mas, com o tempo percebeu-se a possibilidade de utilizá-los para sistematizar os processos e a organização educacional e reestruturar o papel docente (Tajra, 2000).

As TIC permitem a transição do papel professor, de único detentor do saber para mediador no processo de formação possibilitando ao estudante adoção de condutas mais ativas de modo que coautoria, pró-atividade e colaboração são aspectos centrais (Silva 
Neto, 2009). Assim, as TIC modificam os tempos, os ambientes e as formas habituais de nos relacionarmos no contexto sociocultural e educacional criando novas formas de interação, novas formas de acesso ao saber e de construção do conhecimento (Marcondes, 2010).

Conforme Mamede Neves (2008), as TIC promovem a informação, comunicação, interação, colaboração e, em consequência disso, otimizam construção de novos conhecimentos. Nesse contexto, a participação do estudante como ator no processo de construção do seu conhecimento é fundamental, mas o professor deve estar presente para orientar, interpretar e contextualizar as necessidades individuais e grupais.

$\mathrm{O}$ uso das tecnologias na educação tem provocado vários questionamentos a respeito dos métodos de ensino utilizados. Isso, serve de indicador para afirmar que o professor deve ter fluência com as ferramentas tecnológicas sabendo o que elas oferecem em potencial e como podem ser exploradas em diferentes situações educacionais (Valente, 2001).

Diante desse cenário, existe a necessidade de uma epistemologia que explique as peculiaridades da aprendizagem nos espaços virtuais. Para isso, os autores Lévy (1993) e Guatarri (1999) apresentam a teoria da ecologia cognitiva no intuito de levar à adequada compreensão das potencialidades e possibilidades de aprendizagem mediada pelas tecnologias intelectuais. Visto que, conforme esses autores, a ecologia refere-se às relações, interações e diálogos entre organismos dinamizando a relação entre seres humanos e tecnologias nos ambientes de aprendizagem.

A ecologia cognitiva consiste num espaço interativo de cognição individual em que são construídas e reconstruídas as competências cognitivas, em um espaço onde se configuram as diferentes formas de conhecer, pensar e aprender (Guatarri, 1999). Nessa perspectiva, o autor afirma surgir a teoria psicológica ecologia cognitiva para fundamentar o processo de aprendizagem mediada pelas TIC, que por ser tão mais dinâmica e complexa em alguns aspectos, é mister uma teoria específica para a sua compreensão.

Nesse contexto, este estudo teve por objetivo investigar contribuição de práticas pedagógicas no ensino superior que integram recursos das tecnologias de informação e comunicação na mediação do processo ensino-aprendizagem configurador de ecologias cognitivas de estudantes dos Cursos de Graduação em Agronomia, Medicina Veterinária e Tecnologia de Alimentos da Universidade Federal de Santa Maria, Rio Grande do Sul.

\section{Construção Metodológica}

O trabalho foi desenvolvido na Universidade Federal de Santa Maria, na cidade de Santa Maria, RS, Brasil, na disciplina de Educação para Ciências Agrárias mediadas pela Tecnologia da Informação e Comunicação, pertencente ao Centro de Ciências Rurais, nos Cursos de Graduação em Agronomia, Medicina Veterinária e Tecnologia dos Alimentos.

O tipo de pesquisa utilizada foi a exploratória. Como instrumento de coleta de dados foi implementado um questionário tipo survey com questões fechadas para os estudantes. A aplicação do questionário foi no mês de agosto de 2011. O questionário de 14 questões (Quadro 1) levou em consideração os seguintes aspectos: recursos tecnológicos utilizados, interesse dos estudantes, disciplina, motivação, participação, aprendizagem, resultados da utilização dos recursos tecnológicos para o processo 
ensino-aprendizagem. Para cada pergunta, os estudantes possuíam opção afirmativa (sim) ou negativa (não).

Quadro 1 - Questionário aplicado para identificar a influência do uso de tecnologia como mediador motivacional no processo ensino-aprendizagem para os estudantes dos Cursos de Agronomia, Medicina Veterinária e Tecnologia de Alimentos da Universidade Federal de Santa Maria - RS.

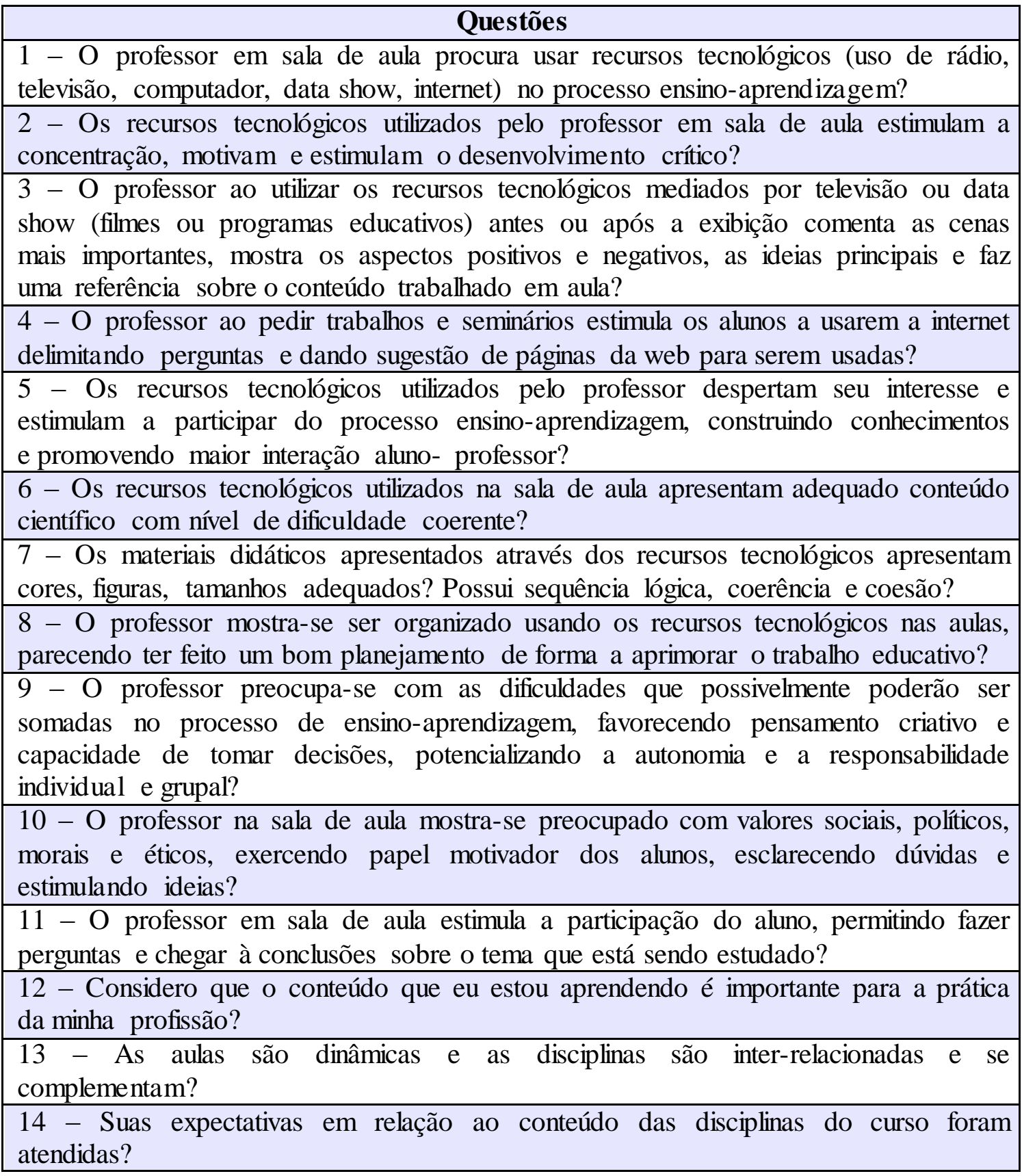

\section{Resultados e Discussão}


A seguir são analisados os indicadores gerados com a aplicação do questionário tipo survey aplicado aos estudantes dos cursos de Agronomia, Medicina Veterinária e Tecnologia de Alimentos da Universidade Federal de Santa Maria. Antes disso, na Figura 1 sistematiza-se graficamente os resultados.

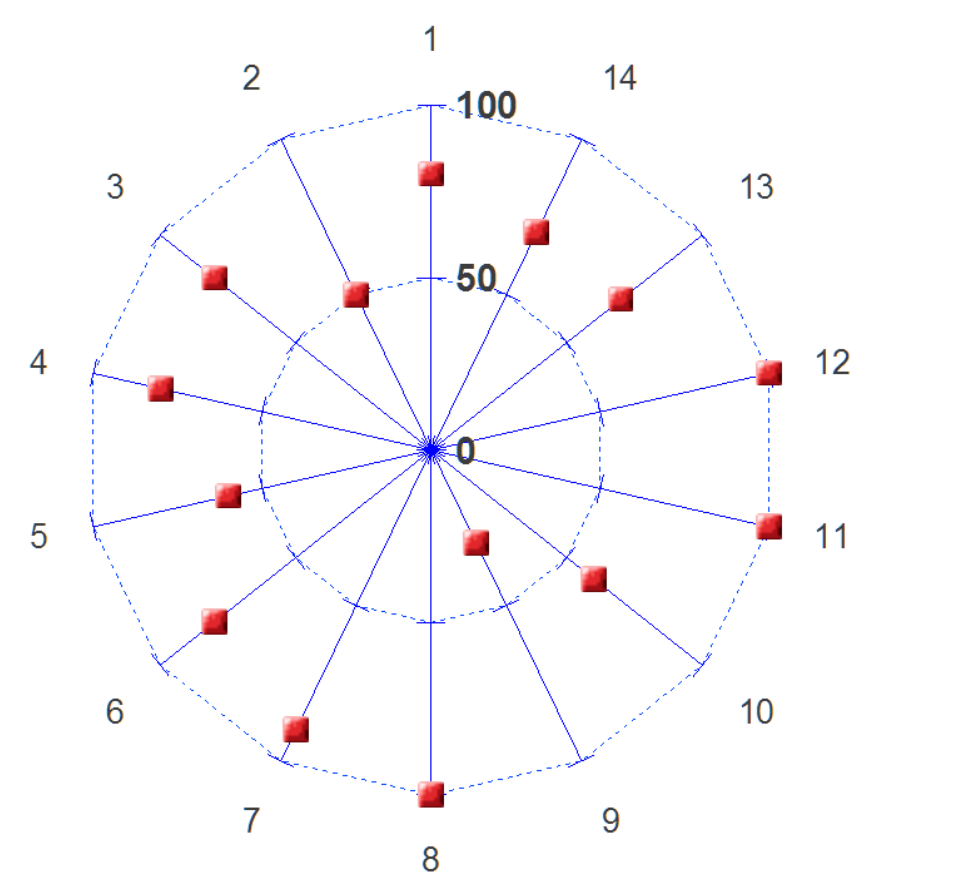

Figura 1 - Rede de resultados dos questionários aplicados aos estudantes de Agronomia, Medicina Veterinária e Tecnologia de Alimentos.

$\mathrm{Na}$ questão 1, 80\% dos estudantes responderam que em sala de aula os professores utilizam recursos tecnológicos. Isso mostra que os professores dos cursos avaliados estão preocupados com a integração dos recursos tecnológicos como forma de estruturar novas metodologias de construção de conhecimento pelos estudantes.

Lévy (1994) ressalta a importância do uso da tecnologia como uma ferramenta de pensamento no sentido em que, ao se articularem com nosso sistema cognitivo, nos ajudam a nos constituir cognitiva e subjetivamente. Segundo essa perspectiva teórica, o sujeito se constrói e se potencializa para novos agenciamentos e aberturas para patamares mais complexos de desenvolvimento em prol de uma ecologia cognitiva coletiva, ajudando na tomada de decisões e na compreensão do mundo e suas circunstâncias. Pois, é no coletivo e com o auxílio das ferramentas tecnológicas (especialmente as hipermídias) que é possível a construção do conhecimento através de autonomia, problematizações, desafios e diálogos promovendo interatividade e interação entre professores e estudantes.

Cerca de $50 \%$ dos estudantes mostraram que os recursos tecnológicos utilizados pelo professor em sala de aula estimulam a concentração, motivam e estimulam o desenvolvimento crítico (Questão 2). Esse resultado reforça a importância do uso das tecnologias em sala de aula na construção do conhecimento dos estudantes, exigindo cada vez mais inovações tecnológicas que busquem motivar e estimular interatividade e interação no processo ensino-aprendizagem mediado tecnologicamente. 
A geração de um novo instrumento de conhecimento, que pode ser definido como uma tecnologia intelectual possibilita, como diz Piaget (1982), do ponto de vista instrumental, construir relações e correspondências novas, e são propriamente estas relações que, ao transformar os objetos e os sujeitos do conhecimento, reconfiguram as bases da ecologia cognitiva (Maraschin; Axt, 2010). Ainda, segundo esses autores, a ecologia cognitiva constitui um espaço de agenciamentos, de pautas interativas, de relações constitutivas, no qual se definem e redefinem as possibilidades cognitivas individuais, institucionais e técnicas, é nesse espaço de agenciamentos que são conservadas ou geradas modalidades de conhecer, de formas de pensar, de tecnologias e de modos institucionais de conhecimento.

O uso dos recursos vídeos, filmes e programas educativos por professores, mostrou ser eficiente no processo ensino-aprendizagem, pois a maioria (80\%) dos estudantes afirmaram um bom aproveitamento nestas aulas (Questão 3). Este indicador resultante do survey aplicado corrobora com Kenski (2010), que afirma que o uso das TIC, sobretudo a televisão e o computador, movimentaram a educação e provocaram novas mediações entre a abordagem do professor, a compreensão do aluno e o conteúdo veiculado.

O uso de recursos tecnológicos pode favorecer a constituição da Mente Coletiva, sendo um processo que transforma cada um de seus participantes na direção de novos conhecimentos e, fundamentalmente de novas atitudes epistemológicas, criando condutas crítico-criativas e autonomia para resolução de problemas e dificuldades específicas (Maraschin; Axt, 2010). Lévy (1994) ressalta que palavras, páginas, imagens, gráficos ou partes de gráficos, sequências sonoras, documentos complexos, que podem eles mesmos ser hipertextos auxiliam na não-linearidade $e$ no desenvolvimento de uma ecologia cognitiva.

A maioria dos estudantes $(80 \%)$ afirmam que o professor ao pedir trabalhos e seminários estimula os estudantes a usarem a internet (Questão 4). Ainda, 60\% dos estudantes confirmaram a premissa (Questão 5) de que o professor pode ser um agente mediador da construção do ensino-aprendizado promovendo a interação. Esses resultados são relevantes do ponto de vista da interação como um dos fundamentos da ecologia cognitiva, pois professor e estudante devem interagir e construir equipes para o desenvolvimento de atividades passando a ser parceiros de um mesmo processo de construção e aprofundamento do conhecimento.

Ainda, esses resultados reforçam o estímulo dos professores com os novos processos educativos de ensino-aprendizagem, na autonomia dos estudantes na busca e construção do seu conhecimento e na mediação das tecnologias. Paulo Freire acredita na educação que considere o estudante construtor do seu conhecimento ao invés de somente reproduzi-los ou memorizá-los. A educação mediada por tecnologias não se limita à transferibilidade, pois só aprende verdadeiramente aquele que se apropria do aprendido, transformando-o em apreendido.

Neste contexto, Pontes (2004) relata à relevância da interação entre todos os integrantes do processo: professores, estudantes, técnicos, equipamentos, redes, pessoas e instituições externas da escola e que se encontram na comunidade ou mesmo em outros países. A interação, nas suas diferentes formas, é um elemento marcante na formação e no trabalho escolar.

Adicionalmente, $80 \%$ dos estudantes revelaram que os recursos tecnológicos utilizados na sala de aula apresentam adequado conteúdo científico com nível de dificuldade coerente (Questão 6) e 90\% dos estudantes afirmaram que os materiais 
didáticos apresentados através dos recursos tecnológicos apresentam cores, figuras e tamanho adequados, com sequência lógica, coerência e coesão (Questão 7). Isso mostra a importância de uma aula bem elaborada, atualizada e com conteúdos coerentes, utilizando os recursos tecnológicos disponíveis, valorizando assim a aula e certamente motivando os estudantes e desafiando-os cognitivamente.

Assim, o professor precisa selecionar os recurso mais apropriados a partir de critérios ou escolhas que viabilizem a integração dos mesmos, pois espera-se que o docente contemple o uso das tecnologias em seu planejamento, explorando-as de forma sábia e funcional, ou seja, não basta apenas ter acesso à esses instrumentos, sobretudo, é preciso saber utilizá-los fluentemente (Lima et al, 2010).

Para utilizar a tecnologia na educação é importante um bom planejamento para obter ganhos no trabalho educativo com os conteúdos escolares (Barros, 2009). Todos os estudantes (100\%) dos Cursos de Graduação estudados, afirmaram que o professor demonstra ser organizado usando os recursos tecnológicos nas aulas e constitui-se um reflexo de um bom planejamento que objetiva a aprimorar o trabalho educativo (Questão 8).

Por outro lado, um fator importante a considerar é que, de acordo com os resultados obtidos, apenas $30 \%$ dos estudantes revelaram que o professor preocupa-se com as dificuldades que possivelmente poderão ser somadas no processo ensinoaprendizagem, problematizando situações e proporcionado materiais que favoreçam o pensamento criativo e a capacidade de tomar decisões, potencializando assim a autonomia e a responsabilidade individual e grupal (Questão 9). Isto reflete uma prática docente tradicional pautada no par transmissão- recepção que pouco se ocupa da necessidade de instigar o estudante a refletir e estabelecer relações entre o conceito $\mathrm{e}$ sua aplicação, demostrando a necessidade de aprimoramento constante por parte do professor para reaprender a ensinar e do aluno a aprender a aprender.

Segundo Kenski (2010), educar para a inovação e a mudança significa planejar e implantar propostas dinâmicas de aprendizagem, em que se possam exercer e desenvolver concepções sóciohistóricas da educação nos aspectos cognitivo, ético, político, cientifico, cultural, lúdico e estético em toda a sua plenitude, e assim, garantir a formação de pessoas para o exercício da cidadania e do trabalho com liberdade e criatividade.

Na Questão 10, 60\% dos estudantes apontaram que os professores na sala de aula mostram-se preocupados com valores sociais, políticos, morais e éticos, exercendo papel motivador, esclarecendo dúvidas e estimulando ideias. Junte-se à isso, todos os estudantes (100\%) (Questão 11 e 12) consideram que os professores em sala de aula desafiam os estudante à participar permitindo a realização de perguntas até chegar à conclusões sobre o tema que está sendo estudado.

Conforme Nascimento (2011), é importante a construção de uma educação integralizadora do ser e do saber, mediante o desenvolvimento de processos educativos que tenham por base uma nova ecologia cognitiva, pautada nos pilares da teoria da complexidade. O conhecimento construído na base da interatividade das hipermídias pode gerar novas visões de mundo, mentalidades abertas e pensamentos reformados, que façam do aprender a aprender e do reaprender a pensar as metas de uma educação emancipatória ao longo da vida, podendo mover atos criativos na direção de uma educação que forme e informe os seres de suas imensas potencialidades cognitivas.

A maioria dos estudantes (70\%) afirmaram que as aulas são dinâmicas e as disciplinas são inter-relacionadas atendendo, assim, as expectativas em relação ao 
conteúdo das disciplinas do curso (Questão 13 e 14). Isso permite um elo para a construção da interdisciplinaridade. A escola precisa garantir aos estudantes (cidadãos) a formação e a aquisição de novas habilidades, atitudes e valores para que possam viver e conviver em uma sociedade em permanente processo de transformação, cidadãos flexíveis o suficiente para incorporar novos e diferenciados perfis profissionais, que tenham consciência da velocidade das mudanças (Kenski, 2010).

\section{Conclusões}

Os resultados da pesquisa survey com foco na integração das tecnologias da informação e comunicação nas práticas pedagógicas do ensino superior evidenciam pontos de inovação didático-metodológica tanto nas aulas presenciais quanto nas atividades extraclasse. A utilização de recursos como vídeos e programas educativos mostra-se eficiente no processo ensino-aprendizagem do ponto de vista da maioria dos estudantes porque os mesmos avaliam que o trabalho pedagógico em torno de conteúdos curriculares torna-se mais dinâmico e interativo.

Com base nos autores referenciados ao longo do texto aponta-se que a integração das tecnologias na educação (essencialmente as de natureza hipermidiática) requer reflexão constante sobre as possibilidades no trabalho pedagógico. Desse modo, a partir dos indicadores gerados no survey, pode-se construir relação da contribuição dessa pesquisa para estudos sobre integração das tecnologias nos cursos de graduação presenciais e a distância da Universidade Federal de Santa Maria.

$O$ uso de recursos da tecnologia da informação e comunicação na mediação pedagógica reflete em maior motivação dos estudantes, dinamizando o processo ensinoaprendizagem, alterando os espaços de ensinar e aprender, modificando a ecologia cognitiva dos sujeitos envolvidos, potencializando o desenvolvimento do raciocínio e possibilitando a aquisição da fluência tecnológica e a autonomia do aprendizado.

Com base nos resultados obtidos, pode-se afirmar que o uso das tecnologias no ensino-aprendizado requer do professor performance, preocupada não apenas no repasse de conteúdos tão comum na educação bancária, mas na transformação do estudante de passivo para sujeito do processo de aprendizado.

A integração de TIC nos Cursos de Graduação estudados promove um despertar para a transição do ensino baseado na transferência de conteúdos para um ensino mais autônomo, dinâmico, não-linear e pró-ativo. Estimula o estudante à desenvolver a ecologia cognitiva individual em prol de uma coletiva.

A integração de recursos das tecnologias de informação e comunicação modifica a concepção de ensino-aprendizagem gerando interatividade e interação mais contínua e sensorial. Além da verbalização, a hipermídia potencializa interatividade que produz interação e criação de conhecimento. O processo ensino-aprendizagem mediado por hipermídia permite aliar o conhecimento e a formação epistemológica à integração reflexiva, pedagógica, coletiva, articuladora e atrativa dos recursos configurando a ecologia cognitiva. Assim, a interatividade resignifica a relação indivíduo-objeto criando uma nova dinâmica nos processos de construção do saber baseados na existência de relações, diálogos e interações.

\section{Referências Bibliográficas}

ALVES. L.; NOVA, C. Educação a distancia: uma nova concepção de aprendizado e interatividade. São Paulo: Futura, 2003. 
BARROS, D.M.V.B. Guia didático sobre as tecnologias da comunicação e informação:material para o trabalho educativo na formação docente. Rio de Janeiro:Vieira \& Lent, 2009. 160p.

GONÇALVES, B. L. V.; MARCONDES, C. H. O impacto da Internet nos serviços bibliotecários: um estudo exploratório. In: SEMINÁRIO NACIONAL DE BIBLIOTECAS UNIVERSITÁRIAS, 10., 1998, Fortaleza. Anais. Fortaleza.1998. (CD-ROM).

GUATARRI, F. As três ecologias. Campinas: Papirus. 1999. 56 p.

KENSKI, V.M. Educação e tecnologias o novo ritmo da informação. Campinas: Papirus. 2010. $141 \mathrm{p}$.

LÉVY, P. As tecnologias de inteligência: o futuro do pensamento na era da informática. Rio de Janeiro: Editora_34, 1993. 208 p.

LÉVY, P. As Tecnologias da Inteligência. Nova Fronteira: Editora 34. 1994.

LIMA, S.A.; ALMEIDA; M.P.; RESENDE, L.M.M. Investigar a prática pedagógica: uma contribuição à formação de professores de ciências e matemática. In: SIMPÓSIO NACIONAL DE ENSINO DE CIÊNCIA E TECNOLOGIA, 2., 2010, Ponta Grossa. Anais. Ponta Grossa. 2010. (CD-ROM).

MAMEDE-NEVES, M.A.C.; DUARTE, R. O contexto dos novos recursos tecnológicos de informação e comunicação e a escola. Campinas, v. 29, n. 104 - especial, p. 769789, out. 2008. Disponível em: http://www.cedes.unicamp.br. Acesso em: 15 set. 2011. MARASCHIN, C., AXT, M. O enigma da Tecnologia na Formação Docente. Brasília: Brasil, 1998. Disponível em:

http://webcache.googleusercontent.com/search?q=cache:PaCi6ciKHzkJ:http://www.c5.

cl/ieinvestiga/actas/ribie98/209.html+ecologia+cognitiva++levy+tecnologia\&hl=pt-

BR\&biw=1280\&bih=673\&strip=1 >. Acesso em: 18 de out. 2011.

MARCONDES, C.H. Tecnologias da informação e impacto na formação do profissional da informação. 2010. Disponível em:

http://biblioteconomia.multiply.com/journal/item/5/5?\&show_interstitial=1\&u=\%2Fjou rnal\%2Fitem. Acesso em: jul. de 2011.

MORIJE. J.V.; PAVAN, C. Tecnologias de informação e comunicação: novas sociabilidades nas bibliotecas universitárias. Ciência da Informação, V. 33, n. 1. 2004. MORTIMER, E.F.: SCOTT, P. Atividade discursiva nas salas de aula de ciências:uma ferramenta sociocultural para analisar e planejar o ensino. Investigações em Ensino de Ciências. Porto Alegre, v.7 n.3. 2002. Disponível em:

<http:/www.if.ufrgs.br/public/ensino/vol7/n3/v7_n3_a7.htm>. Acesso em:21 set. 2011. NASCIMENTO, R.N.A., A complexidade como matriz de uma nova ecologia cognitiva. Paraîba. João Pessoa: UFPB/PB, 2007. 284p. Tese de Doutorado.

PERRENOUD, P. Dez novas competências para ensinar. Porto Alegre: Artes Médicas, 2000.

PESSANHA, R.F. Recursos Tecnológicos e Educação: Amplitude de Possibilidades. 2009. Disponível em: http://www.pedagogia.com.br/artigos/tecnologia/. Acesso em: out. 2011.

PIAGET, Jean. O nascimento da inteligência na criança. Rio de Janeiro: Zahar,1982. PONTES, B.R. Planejamento, Recrutamento e Seleção de Pessoal ( $4^{a}$ ed.). São Paulo: LTR, 2004.

SILVA, K.F.; SILVA NETO, S.A. O processo de ensino aprendizagem apoiado pelas TIC's: repensando práticas educacionais. 2009. Disponível em: http://ketiuce.hdfree.com.br/TDAE/Artigo_Ketiuce.pdf. Acesso em: jul. de 2011. 
SIMÕES, V.A.P. Utilização de novas tecnologias educacionais nas escolas da rede estadual da cidade de Umuarama - PR. Uberlândia: UFU, 2002. 100p. Dissertação de Mestrado em Educação.

TAJRA, S.F. Informática na Educação: novas ferramentas para o professor da atualidade. $2^{a}$ ed. São Paulo: Érica, 2000.

VALENTE, J. O computador como ferramenta educacional. 2001. Disponível em: $<$ http://www.nied.unicamp.br/publicacoes/separatas/sep4.pdf $>$. Acesso em: set. de 2011. 